\title{
Chiral DNA origami nanotubes with well-defined and addressable inside and outside surfaces
}

\author{
Florence Benn, Natalie E. C. Haley, Alexandra E. Lucas, Emma Silvester, Seham Helmi, Robert \\ Schreiber, Jonathan Bath and Andrew J. Turberfield*
}

\begin{abstract}
We report the design and assembly of chiral DNA nanotubes with well-defined and addressable inside and outside surfaces. We demonstrate that the outside surface can be functionalised with a chiral arrangement of gold nanoparticles to create a plasmonic device and that the inside surface can be functionalised with a track for a molecular motor allowing transport of a cargo within the central cavity.
\end{abstract}

An important application for DNA nanostructures is the provision of scaffolds that precisely control the numbers and positions of functional molecules: DNA nanostructures can be used as containers for a payload ${ }^{1,2,3}$ or as test platforms for singlemolecule chemistry ${ }^{4}$ or enzyme assays. ${ }^{5}$ DNA nanostructures have also been used to study enzyme cascades by controlling the relative positions of two or three enzymes. ${ }^{6,7}$ Encapsulating the enzyme cascade within a tube provides a way to control the local environment. Fu et al. $^{7}$ tested such a system based on a rectangular DNA origami tile $^{8}$ rolled up to generate a mixed population of tubes, of which some contained the enzymes on the inside and some displayed them on the outside. It is with this application in mind that we designed an addressable DNA nanotube with well-defined inside and outside surfaces.

Systems designed to form $2 \mathrm{D}$ arrays from discrete tiles often produce tubular structures. ${ }^{9,10,11,12,13}$ In such cases there is little control over the diameter of the tube. Formation of tubes can be discouraged by arranging the component tiles so that their intrinsic curvatures cancel ${ }^{9,11,12}$ or by reducing the flexibility of the tile. ${ }^{11}$ Formation of tubes can be encouraged by deliberately introducing curvature to give tubes with a well-defined diameter and specific inside/outside surfaces. ${ }^{14,15}$ Selective insertion/deletion of base pairs ${ }^{16}$ allows the assembly of nanotubes with a defined chirality. ${ }^{15}$

Tubes can also be formed by joining opposite edges of a DNA origami ${ }^{8}$ rectangle. ${ }^{7,17}$ This approach creates a fully addressable surface, that allows control of the identities, numbers and positions of functional groups attached to the oligonucleotide 'staples' that weave through the structure, but it produces a mixture of inside-out and right-side-out tubes. Here we introduce curvature into DNA origami to form addressable chiral tubes with defined inside/outside surfaces. To demonstrate that the tubes assemble as designed, we decorate the outside of the tube with a chiral arrangement of gold nanoparticles and measure the characteristic circular dichroism (CD) signal. ${ }^{18}$ We then link two tubes whose inside surfaces are decorated with the track for a molecular motor and demonstrate that a molecular cargo can be transported the length of the track inside the tubes.

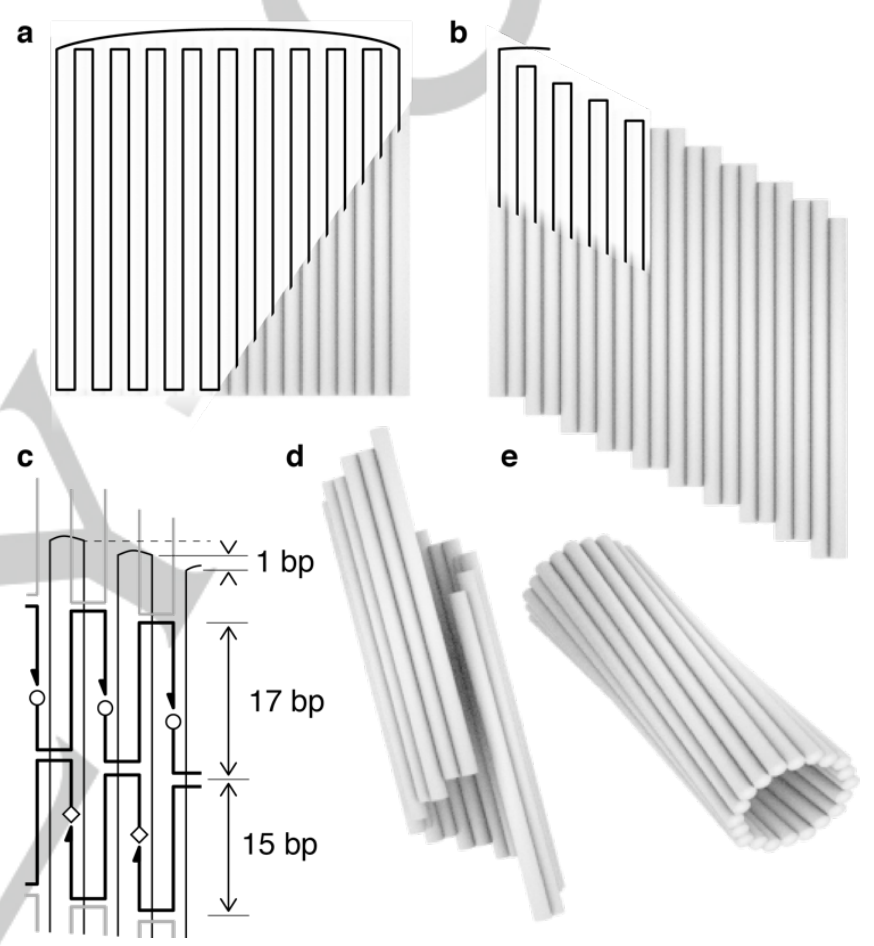

Figure 1. Design of a chiral DNA nanotube. (a) The design is based on a rolled sheet of origami with 20 helices, each linked to its neighbours by both staple and scaffold crossovers. There is no central seam and no edges: the first and last helices are linked directly by staples and template. (b) Pairs of helices are translated by 1 bp by systematically translating staple crossover positions as indicated in panel c. Staples with a circle at the $5^{\prime}$ end have an 8-16-9 nt domain structure $\left(5^{\prime}\right.$ to $\left.3^{\prime}\right)$, staples with a square at the $5^{\prime}$ end have an 8-15-8 nt domain structure; $3^{\prime}$ ends of bold staples are indicated with an arrowhead. Grey staples have $T_{10}$ tails to block interaction between tubes (d) The relative displacement of the crossovers to the left and right of each helix introduces curvature (the diagram shows the split tube that would result if all connections between two adjacent helices were cut). (e) The seamless tube assembles with a shallow right-handed twist which brings the edges of the notional split tube shown in panel $\mathbf{d}$ into register, allowing all connections between helices to be made. The figure is drawn to illustrate design, not assembly ( $\mathbf{a}, \mathbf{b}$ and $\mathbf{d}$ do not represent part-folded structures).

Rather than roll up a flat sheet of origami ${ }^{7}$ or twist an origami ribbon ${ }^{16}$ to form a tube, we designed a seamless structure with no top or bottom edge (Supplementary Fig. S1). The design consists of 20 helices that run the length of the tube $(120 \mathrm{~nm})$ and are twisted around the axis of the tube with a shallow right-handed helical pitch (Fig. 1). Each helix is linked to its neighbours around the circumference of the tube by both scaffold and staple 
crossovers. The tube is designed to fold in a single step but it is convenient to describe the design stepwise, starting with a flat sheet of origami with 20 helices (Fig. 1a).

The sheet is curved by changing the spacing between staple crossovers, connecting adjacent helices on either side, from 16 bp $\left(\sim 1 \frac{1}{2}\right.$ turns) to an alternating pattern of $17 \mathrm{bp} / 15 \mathrm{bp}$ (Fig. 1c). This modification translates neighbouring pairs of helices by one base pair $(0.34 \mathrm{~nm})$ along the long axis of the tube (Fig 1b) and rotates them about the long axis by $\sim 34^{\circ}$. After 10 steps an approximate full turn is made. Fig. 1d shows the tube-like structure that would result if all connections (staple and scaffold crossovers) between two adjacent helices were cut to create disconnected edges: the top edge is translated by $3.4 \mathrm{~nm}$ (10 bp) relative to the bottom edge. The shallow right-handed helical pitch of the tube is introduced when the edges are brought back into register and joined ${ }^{14}$ (Fig. 1e) (in reality, they are never separated). The inside and outside surfaces are defined by the systematic offset between staple crossovers to the left and to the right that determines the direction in which the sheet of helices rolls up to form a tube. The surfaces of the tube can be functionalized by modifying extended staples which protrude from the origami at nicks in the DNA backbone where adjacent staples meet; the positions of the nicks can be adjusted such that they lie on the inside or outside surface. ${ }^{19}$ Tubes can be joined end-toend by the inclusion of special linking end staples or the ends of the tube can be passivated to prevent stacking between tubes by adding $T_{10}$ tails to the end staples (Fig. 1c).
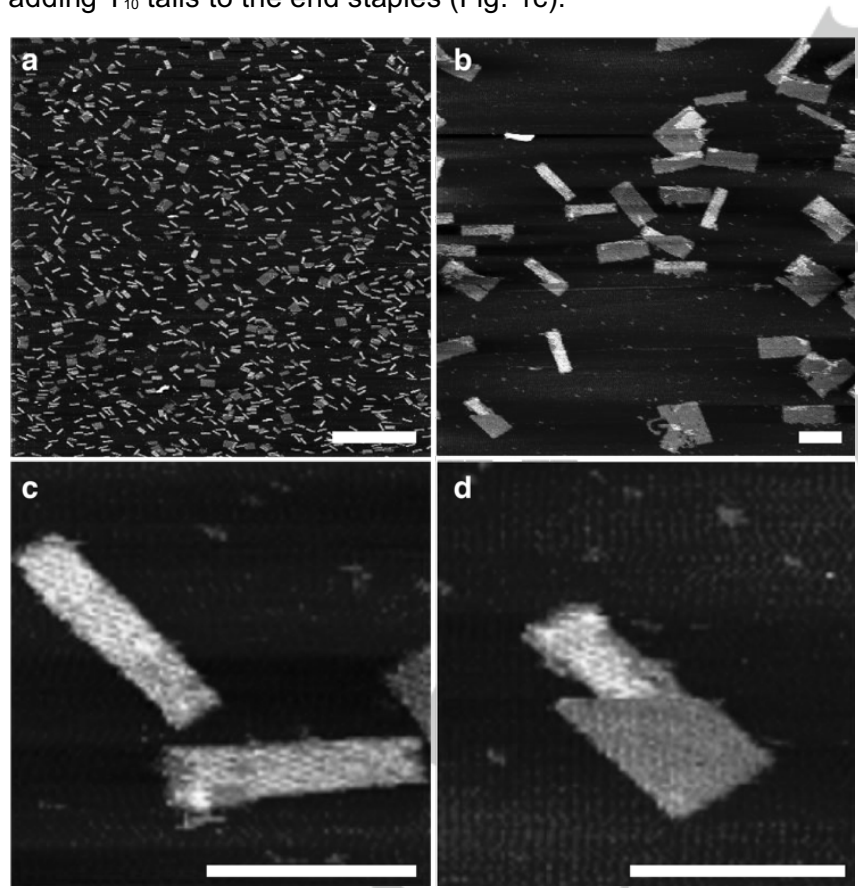

Figure 2. AFM of chiral DNA nanotubes. (a) The majority of objects imaged by AFM are rectangular structures with dimensions of $\sim 120 \mathrm{~nm} \times 30 \mathrm{~nm}$ consistent with tubes that were flattened when deposited on mica. (b) Halfheight rectangular structures with dimensions of $\sim 120 \times 50 \mathrm{~nm}$ are also visible, particularly at higher magnification. These structures appear to be tubes that have been split open. c) High-resolution images reveal the DNA origami lattice structure (d) Tubes can be split open mechanically by increasing the tip force (this tube is partially split open). Scale bars are $1 \mu \mathrm{m}$ (a) and $100 \mathrm{~nm}$ (b-d).

Tubes were assembled in a single step by mixing template, staples and end staples in a ratio of 1:3:5 in a buffer containing
$10 \mathrm{mM} \mathrm{MgCl}, 50 \mathrm{mM} \mathrm{NaCl}, 1 \mathrm{mM}$ EDTA and $10 \mathrm{mM}$ Tris- $\mathrm{HCl}$ $\mathrm{pH} 8.0$ then cooling from $96^{\circ} \mathrm{C}$ to $20^{\circ} \mathrm{C}$ at a rate of $1^{\circ} \mathrm{C}$ per minute (Supplementary Fig. S3). Excess staples were removed by three passes through a spin column packed with Sephacryl S-300 ${ }^{19}$ (Fig.2 and Fig.4) or by PEG precipitation (Fig. 3).

Well-folded rectangular structures were observed by atomic force microscopy (AFM) of samples deposited on mica (Fig. 2). Images are dominated by two types of rectangular structure: double-height rectangles of $\sim 120 \mathrm{~nm} \times 30 \mathrm{~nm}$ corresponding to flattened tubes, and single-height rectangles of $\sim 120 \mathrm{~nm} \times 50 \mathrm{~nm}$ that have the appearance of tubes that have split open, perhaps when deposited on the mica surface. Consistent with this interpretation, tubes can easily be split open during imaging by increasing the tip force (compare high-resolution images in Fig. $1 \mathrm{c}$ and $\mathrm{d}$ ). It is difficult to estimate the yield of well-folded tubes because it is not clear to what extent they are damaged by deposition on mica and subsequent imaging. However, $66 \%$ (416 of 628 objects counted in Fig 2a) are double-height structures of the correct size. The stability of the tubes could be increased by enzymatic ${ }^{19}$ or chemical ${ }^{20}$ ligation of neighbouring staples.
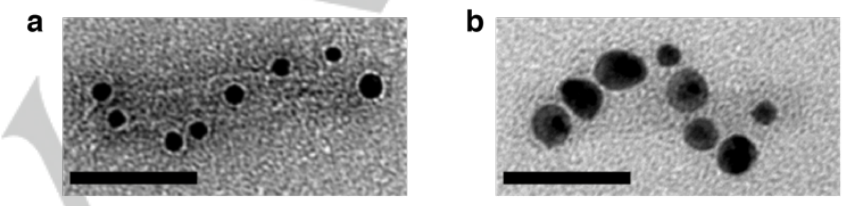

C

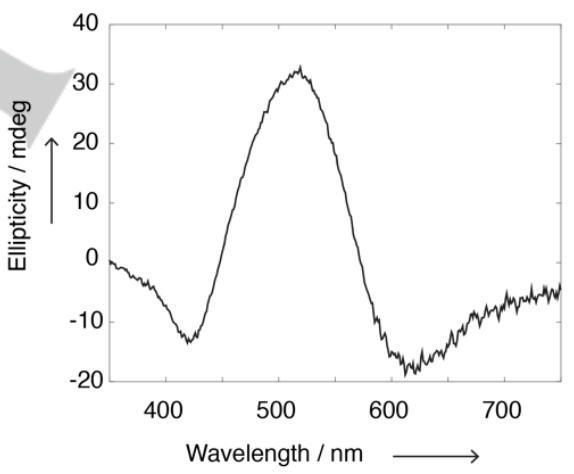

Figure 3. Circular dichroism of chiral DNA nanotubes. (a) The outside surface of the nanotube was decorated with gold nanoparticles in a left-handed helical arrangement and imaged with negative-strain transmission electron microscopy. (b) Prior to circular dichroism (CD) silver was deposited on the gold to enhance the signal (Supplementary Information). Helical structures after enhancement were sparse on e.m. grids when compared with a sample before enhancement. Scale bar in $\mathbf{a}$ and $\mathbf{b}$ is $50 \mathrm{~nm}$. (c) The CD signal for the silverenhanced nanotubes is characteristic of a left-handed helix of nanoparticles.

DNA nanostructures can be functionalised with gold nanoparticles to create structures with a distinct circular dichroism (CD) signals: a left-handed helical array of gold nanoparticles gives a 'peak-dip' signal as wavelength increases whereas a righthanded helix gives a 'dip-peak' signal. ${ }^{18}$ In order to confirm that our tubes assemble right-side out, with the designed chirality, we functionalised the outside of our structures with a left-handed arrangement of nine gold nanoparticles each with a diameter of $10 \mathrm{~nm}$ (Fig. 3a, b), silver was then deposited on the gold to enhance the signal. ${ }^{18}$ The $C D$ signal that we observe is characteristic of a left-handed helical arrangement (Fig. 3c). 
A similar experiment was performed with gold attachment sites on the inside surface of the tube. We anticipated that the relatively small internal diameter of the tube would prevent assembly of DNA functionalised gold nanoparticles inside the tube. ${ }^{22}$ We observe structures that are decorated with gold on the outside of the tube consistent with threading ${ }^{23}$ of the gold attachment sites through the DNA origami and capture on the outside surface by the functionalised gold (Supplementary Fig. S5). These structures, as expected, also have a CD signal characteristic of a left-handed helix (Supplementary Fig. S6).

a
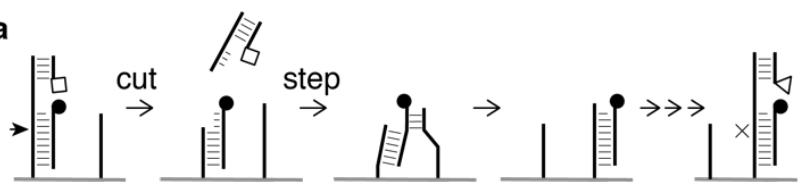

$\diamond=$ TET $\triangle=$ Cy5

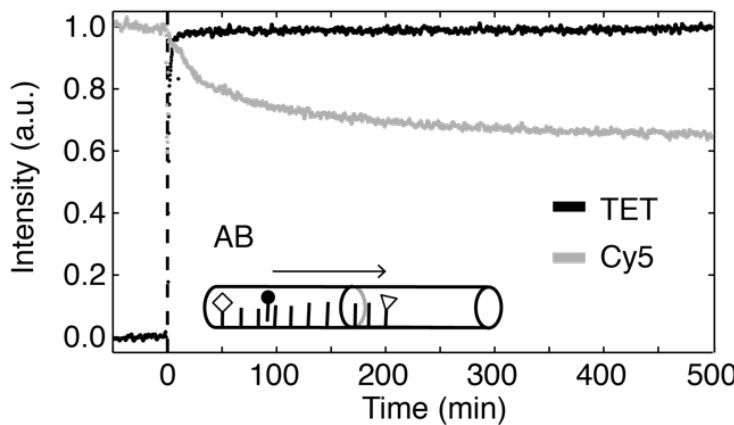

C

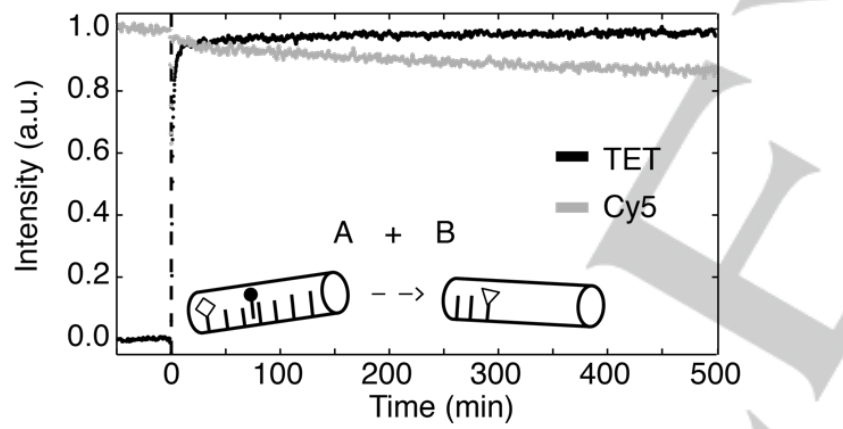
Figure 4. Movement of a molecular motor along a track inside the tube. (a)
The inside of the tube is functionalised with a track of single-stranded anchorages. The track consists of 26 anchorages divided between two tubes $A$ and $B$. The first anchorage in tube $A$ and the last anchorage in tube $B$ are labelled with fluorophores TET (square) and Cy5 (triangle), respectively. The cargo caries the quencher lowa Black RQ (filled circle). The TET signal is quenched when the motor is loaded onto the track at the first anchorage in tube A. Addition of a nicking enzyme ( $\mathrm{Nt.BbvCl}$, cut site marked with an arrowhead) triggers movement of the motor along the track resulting in an increase in TET signal. When the motor reaches the last anchorage in tube B the Cy5 signal is quenched (mismatch preventing nicking marked with an $X$ ). (b) When two tubes $A$ and $B$ are joined together the motor travels the length of the track. (c) When tubes $A$ and $B$ are not joined, arrival of the motor at the end of the track is slower because it requires the motor to move between disconnected tubes.

When the end staples are modified with $T_{10}$ tails there is very little evidence of end-to-end stacking of tubes (Fig. 2). Some applications require two or more tubes to be linked together in a specific register and/or two different tube types to be linked together. We have joined pairs of tubes to create extended tracks for a synthetic molecular motor.
Two separately prepared sets of tubes $A$ and $B$ were joined end-to-end, in register, by introducing linking staples (Supplementary Fig. S2) and the inside surface was functionalised with a track for the 'burnt bridges' molecular motor. ${ }^{19,24,25}$ The track (Fig. 4a) consists of 21 anchorages (staple extensions) in tube $\mathrm{A}$ and a further 5 anchorages in tube $\mathrm{B}$. The start and end anchorages are labelled at the distal end with fluorophores (TET and Cy5 respectively); the cargo carries a quencher (lowa Black RQ). Movement of the cargo is initiated by addition of a nicking enzyme (Nt.BbvCl). The nicking enzyme cuts the anchorage that is bound to cargo. Release of the cut anchorage fragment allows the cargo to step to the next intact anchorage. The position of the cargo on the track is reported by fluorescence: when the fluorophore-labelled start anchorage is cut the fluorophore is released from the track giving an increase in the fluorescence signal as it moves away from the quencherlabelled cargo. Modification of the enzyme recognition site in the end anchorage ${ }^{23}$ prevents cutting and traps the cargo, quenching the corresponding fluorescence signal. The TET signal increases and the Cy5 signal is quenched when movement of the cargo away from the start position is initiated (Fig. 4b), consistent with movement of the motor from one end of the track to the other. The rate at which the motor moves is similar to published results for movement along the surface of a DNA origami tile. ${ }^{19}$ Control experiments where the $A$ and $B$ tubes are not linked show that the cargo is significantly slower to arrive at the end of the B tube (Fig. 4c), consistent with the measured rate for stepping between two unconnected DNA origami tiles in solution. ${ }^{18}$

We have demonstrated one-step assembly of chiral DNA origami nanotubes. The tubes form right-side-out, the inside and outside surfaces can be functionalised, and tubes can be joined together in register. Our chiral nanotubes provide the controlled geometry required, for example, to allow the study of reactions in confined volumes.

\section{Experimental Section}

DNA design (using NUPACK ${ }^{26}$ and caDNAno $^{27}$ ), sequences and experimental detail including PEG purification ${ }^{28}$ are provided in the Supporting Information.

\section{Acknowledgements}

This research was supported by Biotechnology and Biological Sciences Research council grant BB/J00054X/1 and Engineering and Physical Sciences Research Council grant EP/F500394/1. AJT was supported by a Royal Society Wolfson Research Merit Award.

Keywords: DNA nanotechnology $\cdot$ DNA self-assembly $\bullet$ chiral nanotube $\cdot$ plasmonic device $\cdot$ molecular motor

1 C. M. Erben, R. P. Goodman and A. J. Turberfield, Angew. Chem. Int. Ed. 2006, 45, 7414; Angew. Chem. 2006, 118, 7574

2 P. K. Lo, P. Karam, F. A. Aldaye, C. K. McLaughlin, G. D. Hamblin, G. Cosa and H. F. Sleiman, Nat. Chem. 2010, 2, 319.

3 S. M. Douglas, I. Bachelet and G. M. Church, Science 2012, 335, 831. 
$4 \quad$ N. V. Voigt, T. Tørring, A. Rotaru, M. F. Jacobsen, J. B. Ransbæk, R Subramani, W. Mamdouh, J. Kjems, A. Mokhir, F. Besebacher and K. V. Gothelf, Nat. Nanotech. 2010, 5, 200.

5 M. Endo, Y. Katsuda, K. Hidaka and H. Sugiyama, J. Am. Chem. Soc. 2010, 132, 1592

6 O. I. Wilner, Y. Weizman, R. Gill, O. Lioubashevski, R. Freeman and I. Willner, Nat. Nanotech. 2009, 4, 249.

7 J. Fu, M. Liu, Y. Liu, N. W. Woodbury and H. Yan, J. Am. Chem. Soc. 2012, 134, 5516

8 P. W. K. Rothemund, Nature 2006, 440, 297.

9 H. Yan, S. H. Park, G. Finkelstein, J. H. Reif and T. H. LaBean, Science 2003, 301, 1882

10 J. C. Mitchell, J. R. Harris, J. Malo, J. Bath and A. J. Turberfield J. Am Chem. Soc. 2004, 126, 16342.

11 W. Liu, H. Zhong, R. Wang and N. C. Seeman, Angew. Chem. Int. Ed. 2011, 50, 264; Angew. Chem. 2011, 123, 278.

12 G. Tikhomirov, P. Petersen and L. Qian, Nat. Nanotech. 2017, 12, 251.

13 P. Yin, R. F. Hariadi, S. Sahu, H. M. T. Choi, S. H. Park, T. H. LaBean and J. H. Reif, Science 2008, 321, 824.

14 P. W. K. Rothemund, A. Ekani-Nkodo, N. Papadakis, A. Kumar, D. Kuchnir Fygenson and E. Winfree, J. Am. Chem. Soc. 2004, 126, 16344

15 A. M. Maier, W. Bae, D. Schiffels, J. F. Emmerig, M. Schiff and T. Liedl, ACS Nano, 2017, 11, 1301.

16 H. Dietz, S. M. Douglas and W. M. Shih, Science 2009, 325, 725.

17 M. Endo, S. Yamamoto, T., T. Emura, K. Hidaka, N. Morone, J. E. Heuser and H. Sugiyama, Angew. Chem. Int. Ed. 2014, 53, 7484; Angew. Chem, 2014, 126, 7614

18 A. Kuzyk, R. Schreiber, Z, Fan, G. Pardatscher, E. -M. Roller, A. Högele, F. C. Simmel, A. O. Govorov and T. Liedl, Nature 2012, 483, 311.

19 S. J. Wickham, M. Endo, Y. Katsuda, K. Hidaka, J. Bath, H. Sugiyama and A. J. Turberfield, Nat. Nanotech. 2011, 6, 166.

20 P. O'Neill, P. W. K Rothemund, A. Kumar and D. K. Fygenson, Nano Lett. 2006, 6, 1379.

21 V. Cassinelli, B. Oberleitner, J. Sobotta, P. Nickels, G. Grossi, S. Kempter, T. Frischmuth, T. Liedl and A. Manetto, Angew. Chem. Int. Ed. 2015, 54, 7795; Angew. Chem. 2015, 54, 7795.

22 W. J. Parak, T. Pellegrino, C. M. Mucheel, G. Geriom, S. C. Williams and A. P. Alivisatos, Nano Lett. 2003, 333.

23 N. Wu, D. M. Czajkowsky, J. Zhang, J. Qu, M. Ye, D. Zeng, X. Zhou, J. Hu, Z. Shao, B. Li and C. Fan, J. Am. Chem Soc. 2013, 135, 12172.

24 J. Bath, S. J. Green and A. J. Turberfield, Angew. Chem. Int. Ed. 2005,44, 4358; Angew. Chem. 2005, 117, 4432.

25 S. F. J. Wickham, J. Bath, Y. Katsuda, M. Endo, K. Hidaka, H. Sugiyama and A. J. Turberfield, Nat. Nanotech. 2012, 7, 169.

26 J. N. Zadeh, C. D. Steenberg, J. S. Bois, B. R. Wolfe, M. B. Pierce, A. R. Khan, R. M. Dirks and N. A. Pierce, J. Comput. Chem. 2011, 32, 170.

27 S. M. Douglas, A. H. Marblestone, S. Teerapittayanon, A. Vasquez, G. M. Church and W. M. Shih, Nucleic Acids Res. 2009, 37, 5001.

28 Stahl, E., Martin, T. G., Praetorius, F and Dietz, H, Angew. Chem. Int. Ed. 2014 53, 12735 ; Angew. Chem. 2014, 126, 12949. 


\section{COMMUNICATION}

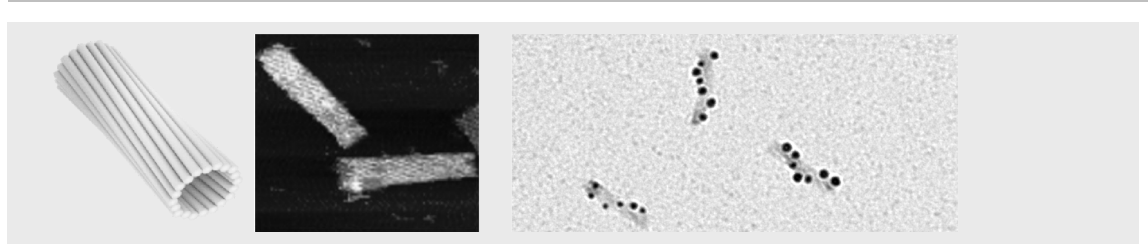

We report the design and assembly of chiral DNA nanotubes with well-defined and addressable inside and outside surfaces.
Florence Benn, Natalie E. C. Haley, Alexandra E. Lucas, Emma Silvester, Seham Helmi, Robert Schreiber, Jonathan Bath and Andrew J. Turberfield*

Page No. - Page No.

Title 